\title{
Efficacy of Fiber Glass Cast in Treatment of Long Bone Fractures in Clinical Cases of Dogs
}

\author{
Adarsh Bijapur, G.U. Yadav* and D.U. Lokhande
}

Department of Veterinary Surgery \& Radiology, Bombay Veterinary College, Parel, Mumbai-12, Maharashtra Animal and Fishery Sciences University, Nagpur (M.S), India

*Corresponding author

\begin{abstract}
A B S T R A C T

\begin{tabular}{|l|}
\hline Key w o r d s \\
Dogs, Fiber glass \\
cast, Long bone \\
fracture
\end{tabular}

\section{Introduction}

Fracture is defined as the break in the continuity of a hard tissue including bone and cartilage. Fractures in dogs are caused due to automobile accident, fall from height, dog bites or kicking by the other animals. Long bone fractures are treated by different methods of immobilization like external fixation, internal fixation and immobilization by casting, bracing, or splinting. External cooptation technique doesn't disturb internal inflammatory process which favours quicker fracture healing. Numbers of synthetic materials are now days are available for
\end{abstract}

Six dogs suffering from long bone fractures were immobilized under general anesthesia by using commercially available fiber glass cast. Automobile accident was recorded as cause for long bone fracture in 5 dogs $(83.33 \%)$ while fall from height was recorded in 1 (16.6 $\%)$ dogs. 3 cases $(50 \%)$ were diagnosed as simple oblique fractures and 3 cases $(50 \%)$ as simple transverse fractures. Propofol provided adequate depth of anaesthesia and smooth recovery in young dogs less than 6 months of age. Immobilization with of fiberglass cast was easy and less time consuming. Method of application was simple and less cumbersome. Fiberglass cast attained its toughness i.e. in around 5-8 minutes. Fiberglass cast was found radiolucent, lighter in weight, stronger and durable compared to a conventional plaster of paris. Few of the complications such as muscular atrophy, excoriation on skin and swelling were noted during the study.

casting which have excellent conforming characteristics such as fiberglass.

\section{Materials and Methods}

The present study was conducted on 6 young dogs suffering from long bone fractures. Each dog was examined thoroughly for the site, type, duration of fracture, local inflammatory sign and displacement of fractured bone. The radiographs of affected limbs were taken in anterio-posterior and medio-lateral position for each case before and after immobilization, $30^{\text {th }}$ day and $60^{\text {th }}$ day of post immobilization. All the dogs having long bone fractures were 
immobilized with fiberglass casts under general anesthesia. Dexamethasone and atropine sulphate were used as a preanesthetic in all the dogs at the dose rate of $0.04 \mathrm{mg} / \mathrm{kg}$ body weight and $0.5 \mathrm{mg} / \mathrm{kg}$ body weight respectively. Each case was sedated with triflupromazine $(1 \mathrm{mg} / \mathrm{kg}) \mathrm{I} / \mathrm{V} 5$ minutes prior to induction with general anaesthesia. After sedation, either thiopentone sodium $(12.5 \mathrm{mg} / \mathrm{kg})$ or propofol $(3-4 \mathrm{mg} / \mathrm{kg})$ were used as general anaesthetics for fiberglass casting. In the present study, fiberglass casts ${ }^{7}$ of different sizes ( 2 inch, 3 inch and 4 inch) were used for the immobilization of fracture according to the size of dog. The dog was placed in lateral recumbancy with affected limb upside under general anaesthesia. This was reduced by applying traction and counter traction maintaining normal angulations of limb. Sufficient quantity of cotton was applied on limb specifically on pressure points including two joints one proximal and one distal to fracture site and the gauze bandage was rolled over it. Fiberglass cast was immersed in the water having temperature of $20-25^{0} \mathrm{C}$ it for 10 seconds. The fiberglass cast was rolled over fractured limb in layers, each layer covering the $50 \%$ of the preceding layer covering two joints, one proximal and one distal to fracture site. Fiberglass was allowed to set for 10-15 minutes. The clinical parameters like age of affected dogs, site of fracture, weight bearing, gait, cost of implant and radiological study were performed.

\section{Results and Discussion}

Out of six dogs with long bone fracture, 4 cases $(66.66 \%)$ were found below one year of age, and 2 cases $(33.33 \%)$ were of above one year of age. Automobile accident was recorded as cause for long bone fracture in 5 dogs $(83.33 \%)$ while fall from height was recorded in $1(16.6 \%)$ dogs. Similar findings have also been noted by Virkar (1999),
Abdulrahman et al., (2003). In the present study out of six animals, 3 animals (50\%) were diagnosed with fracture of fore limb and 3 animals $(50 \%)$ with fracture of hind limb. Aithal et al., (1999) observed fracture more frequently in fore limb $(66.67 \%)$ than in hind limb (33.33\%). 3 cases (50 \%) were diagnosed as simple oblique fractures and 3 cases $(50 \%)$ as simple transverse fractures. Kushwaha et al., (2011) also reported 57.20\% oblique fractures in canines. Four cases showed mid shaft fractures (66.66\%), one case showed distal third fracture $(16.6 \%)$ and one case $(16.6 \%)$ had fracture in proximal third of the bone. Similar findings were recorded by Aithal et al., (1999).

Preoperatively dogs were kept off food and water for 12 hours. Propofol provided adequate depth of anaesthesia and smooth recovery in young dogs less than 6 months of age but adequate muscle relaxation was not attained while Thiopentone provided good muscle relaxation in dogs more than 6 months age. Hall and Chamber (1987) opined propofol as one of the best Anaesthetic agents in $\operatorname{dogs}$ as it produced quiet and rapid induction of anaesthesia as well as smooth and fast recovery.

Immobilization with of fiberglass cast was easy and less time consuming. Method of application was simple and less cumbersome. Fiberglass cast attained its toughness i.e. in around 5-8 minutes. Solanki et al., (2016) reported that fiberglass sets within 3-5 min. rapid setting of fiberglass cast was also reported by Parmar et al., (2014). On radiographic examination, fiberglass cast was found radiolucent which allowed for radiographic study of fracture healing. Fiberglass cast was lighter in weight, stronger and durable compared to a conventional plaster of paris. Gait of all the dogs were thoroughly studied and recorded on 1st, 10th, 20th, 30th and 60th days post immobilization. 
On 10th day all six dogs exhibited slight weight bearing on immobilized limb. They exhibited moderate lameness but weight bearing was present. On 30th day, 'Excellent' weight bearing was noted in three dogs while remaining 3 dogs attained excellent weight bearing by $60^{\text {th }}$ day. These animals showed complete weight bearing on affected limb without any noticeable lameness. Dogs attained full mobility in $40.67 \pm 4.80$ days. The radiographs of the affected limb in all the cases were taken in anterio-posterior (AP) and medio-lateral (L) view before external immobilization, immediately after immobilization, on 30th and 60th day post immobilization to assess the fracture healing. During radiographic studies fiberglass casts were found radiolucent which facilitated proper visualization of fracture fragments and its healing stages. Solanki et al., (2016) noticed better visualization of fracture fragments in case of fiberglass casts (Fig. 1 and 2).

Fig.1 AP \& ML view of radiograph of right radio-ulna in immediately after external immobilization with fiberglass cast

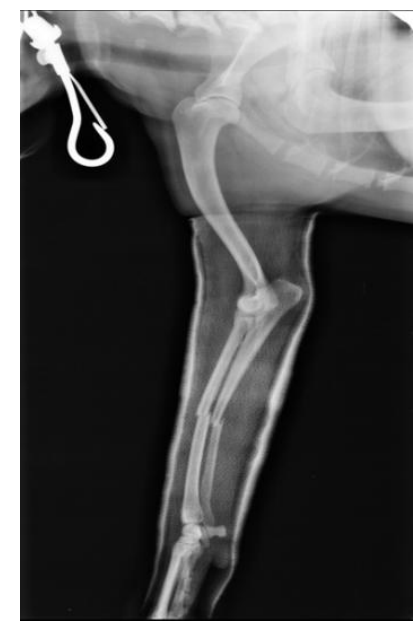

Fig.2 AP \& ML view of radiograph of right radio-ulna on 60th day after external immobilization with fiberglass cast showing dense callus with bridging of cortex formation
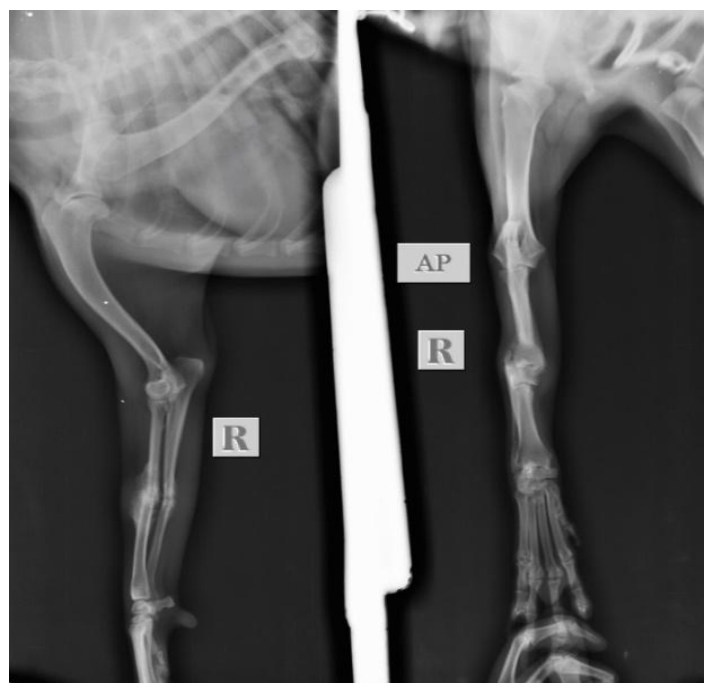
On radiological examination of affected limb before immobilization, fracture line was clearly evident with sharp, well defined loss of radiographic density in all the cases Radiographs also showed soft tissue swelling around the fracture site which may be due to formation of hematoma and inflammation around the fracture site. On 30th day of post immobilization, three cases showed both Periosteal and endosteal reaction with dense callus formation. Fracture line was no longer sharp because of the resorption of the bone along the fracture ends and dense callus formation. There was a decrease in size of fracture line but the fracture line was faintly visible due to development of primary callus at the fracture site. While three cases showed dense callus showing bridging of cortex with calcification. The fracture line was completely invisible in these cases. Patel (2014) reported dense callus, bridging of cortex along and calcification in three cases, periosteal reaction with soft callus in two cases and periosteal and endosteal reaction with dense callus in one case in goats treated with hybrid cast for treatment of long bone fracture. Radiographs on 60th post immobilization day revealed normal radiographic density of dense callus with bridging of cortex. The fracture line and gap was completely absent in all the cases with presence of dense secondary callus. All the animals by 60th day post immobilization exhibited excellent weight bearing supporting the radiographic studies. Singh et al., (2008) reported complete union of the fracture fragments with obliteration of fracture line, ossification and bridging of cortex from 5 th 8th week, post casting in dogs treated with fiberglass cast.

The mean cost of fiberglass cast was Rs. 1050 \pm 89.44 . It was more expensive compared to a conventional plaster. Some of the complications were observed in dogs treated with fiberglass casts. Muscular atrophy and wounds/ excoriations at pressure points were noted in 2 cases. Swelling in paw was seen in 1 case. Similar findings were recorded by Marson and Keenan (1993) and Singh et al., (2008).

\section{References}

Abdulrahman, H. I., A. Mohammed and M. M. Bukar (2003) A retrospective study of fracture cases presented to university of Maiduguri veterinary teaching hospital. Nigerian Veterinary Journal. 27 (3): 25-35.

Aithal, H. P., G. R. Singh and G. S. Bisht (1999) Fractures in dogs: A survey of 402 cases. Indian Journal of Veterinary Surgery, 20(1): 15-21.

Hall, L. W. and J. P. Chamber (1987) A clinical trial of propofol infusion anaesthesia in dogs. J. Small. Anim. Prac. 28: 623-637.

Kushwaha, R. B., A. K. Gupta, M. S. Bhadwal, S. Kumar and A. K. Tripathi (2011) Incidence of fractures and their management in animals: A clinical study of 77 cases. Indian Journal of Veterinary Surgery, 32 (1): 54-56.

Marson, B. M. and M. A. Keenan (1993) Skin surface pressures under short leg casts. Journal of Orthopaedic Trauma, 7(3): 275-8.

Parmar, A. J., S. K. Tyagi, V. S. Dabas, J. N. Mistry, D. N. Suthar, R. H. Bhatt \& I. M. Bhatti (2017) Comparative biomechanical evaluation of plaster of Paris bandage and fibreglass bandage casts under compression testing. Indian Journal of Veterinary Surgery, 38(1): 23-26.

Patel, S. K. (2014). Comparative study on plaster of paris and hybrid cast for long bone fracture in goats (Doctoral dissertation, Nanaji Deshmukh 
Veterinary Science University Jabalpur).

Singh, V., P. R. Dubi and T. K. Gahlot (2008) Clinical study on efficacy of two selected external immobilization technique for long bone fracture repair in goats (Capra hircus). Intas Polivet. 9 (1): 89-96.

Solanki K. P., P. B. Patel, V. D. Dodia, J. V. Vadalia, R. J. Raval, H. M. Padheriya, and M. D. Khatariya (2016) "Comparative effectiveness of plaster of paris and fibre glass casts in the management of long bone fractures in canines." International Journal of Science, Environment and Technology, Vol. 5:5.

Virkar, S. K. (1999) Comparative study on repair of long bone fracture by using polyvinyl chloride mould and Plaster of Paris cast in goat. M.V.Sc thesis (Surgery and Radiology), Konkan Krishi Vidyapeeth, Dapoli.

\section{How to cite this article:}

Adarsh Bijapur, G.U. Yadav and Lokhande, D.U. 2019. Efficacy of Fiber Glass Cast in Treatment of Long Bone Fractures in Clinical Cases of Dogs. Int.J.Curr.Microbiol.App.Sci. 8(06): 114-118. doi: https://doi.org/10.20546/ijcmas.2019.806.015 